schreiten des langwelligsten Absorptionsgebietes erreicht wird. Von besonderem Interesse sind in dieser Richtung auch noch die Rubensschen. Arbeiten seiner letzten Jahre, zum Teil gemeinsam mit Th. Liebisch, in welchen der Verlauf des Brechungsindex im Ultraroten auch für kompliziertere Kristalle für die verschiedenen Schwingungsrichtungen verfolgt wird. Hier waren zum Teil die zum Vergleich mit der Theorie nötigen elektrischen Konstanten noch nicht bekannt und multen daher erst bestimmt werden. Das Gesamtergebnis war auch hier, dal das optische Verhalten mit zunehmender Wellenlänge kontinuierlich in das der elektrischen Wellen ibbergeht.

Etwas anders liegen die Verhältnisse bei den isolierenden Flussigkeiten. Mier zeigen nämlich die meisten auch für die längsten Rubensschen Wärmestrahlen noch nicht den Wert des Brechungindex, wie er sich nach Maxwell aus der statisch gemessenen Dielektrizitätskonstante berechnet. Sie zeigen vielmehr auch hier noch Dispersion. Diese Dispersion, welche sich bis in das Gebiet kurzer elektrischer Wellen fortsetzt, ist durch die Theorie von Debye erklärt als Folge des Dipolcharakters der Moleküle dieser Flüssigkeiten.

Das Gebiet der Gitterpolarisation unterscheidet sich 'von den bisher besprochenen Erscheinungen dadurch, daB hier die Theorie des untersuchten Phänomens noch nicht vollkommen durchgeführt ist. Hier bestatand die Diskrepanz daher nicht zwischen der Folgerung aus der Theorie and dam experimentellen. Befund, sondern in dem Gegensatz zwischen dem, was das Experiment mit elektrischen and mit optischen Wellen ergeben hatte. Für elektrische Wellen hatte Hertz gefunden, daß sie beim Durchgang durch Gitter vollkommen polarisiert werden, und zwar derart, daß ein solches Gitter undurchlässig ist fur elektrische Wellen, deren elektrischer Vektor den Gitterstaben parallel liegt. Im optischen Spektrum hatte $d u$ Bois genau das Gegenteil gefunden.
Daß dieser Widerspruch nur scheinbar ist, ist durch Rubens gemeinsam mit $d u$ Bois in einer Reihe von Arbeiten nachgewiesen worden, welche zeigen, daß auch hier ein kontinuierlicher Utbergang von den optischen $z u$ den elektrischen Wellen besteht. Solange die Wellenlänge klein ist oder von der Größenordinung der Gitterkonstante, tritt der von $d u$ Bois beobachtete Effekt auf; ist sie grob gegen die Gitterkonstante, so zeigen die Wellen das von Hertz gefundene Verhalten. Die langwelligsten Wärmestrahlen von Rubens werden durch feine Drahtgitter bereits vollständig polarisiert.

Zusammenfassend kann man sagen, daß, wenn auch nach den Rubensschen Arbeiten noch ein kleines Wellenlängengebiet zwischen den längsten bekannten Wärmestrahlen und den kürzesten bisher erzeugten elektrischen Wellen übrig bleibt, das Ziel der Rubensschen Untersuchung doch in vollem MaBe erreicht ist. Die Grenze des der Messung zugänglichen Gebietes der Wärmestrahlen ist durch ihn; nämlich bis zu so großen Wellenlängen hinausgeschoben worden, daB diese längsten Wellen bereits in jeder Beziehung die Eigenschaften der elektrischen Wellen zeigen, so daß an ihrer Identität kein Zweifel mehr möglich ist. Dadurch, dab gleichzeitig der kontinuierliche Übergang von den optischen Lichtwellen bis zu den längsten Wärmewellen in bezug auf alle wesentichen Eigenschaften festgestellt ist, ist der elektromagnetischen Lichttheorie die höchste Sicherheit gegeben worden, die für eine Theorie äberhaupt möglich ist. Es verdient darauf hingewiesen zu werden, idab die theoretischen Stützen der elektromagnetischen Licht. theorie, wie die Theorie der Dispersion und der elektro- und magnetooptischen Erscheinungen, ihren Wert als Stützen gerade der Maxwellschen Theorie infolge der neueren Entwicklung dex Quantentheorie zum großen Teil verloren haben. Ohne die Rubensischen Arbeiten wäre die elektromagnetische Lichtheorie anch heute noch eine Hypothese.

\title{
Die Entdeckung der langwelligen Strahlung des Quecksilberdampfes durch Rubens.
}

\author{
Von O.v. Baeyer, Berlin.
}

Heinrich Rubens hat einen großen Teil seiner wissensehaftlichen Arbeit einem Ziel gewidmet, nämlich der Bestätigung der elektromagnetischen Lichttheorie. Wie er, fußend auf den grundlegenden Arbeiten von Heinrich Hertz, Schritt für Schritt neue Beweisstücke für diese Theorie geliefert hat, das schildert er selbst in meisterhaft klarer. Weise in einem Festvortrag, den er am 25. Juni 1917 in der preuBischen Akademie der Wíssenschaften zu Berlin gehalten hat.

Die Maxwellsche elektromagnetische Licht- theorie geht von der Annahme aus, daß elektrisch hergestelltie Wellen, wie wir sie hentzutage in der drahtlosen Telegraphie verwenden, Wärme- und Lichtwellen ein und derselbe Vorgang sind, der sich nur durch die Schnelligkeit der Schwingungen oder, was dasselbe bedeutet, die Größe ider Wellenlänge unterscheidet und deshalb zusammenfassend als elektromagnetische Welle bezeichnet wird. Es müßten: danach auch die optischen Eigenschaften der Körper, also z. B. der Brechungsexponent, das Absorptions-, Re- 
flexions- und Emissionsvermögen der Körper für Wärmestrahlen und für elektrische Wellen gleicherWellenlänge identisch sein. Experimentell läßt sich diese Forderung aber nicht prüfen, da es nicht möglich ist, Wärmestrahlen und elektrische Strahlen gleicher Wellenlänge herzuzustellen. Aber auch für elektromagnetische Wellen verschiedener Wellenlänge ergibt die Theorie einfache Beziehungen zwischen den optischen und den elektrisehen Konstanten der Körper. Diese Beziehungen hat Rubens geprüft, zuerst mit Wärmestrahlen von $1,25 \mu$ Wellenlänge später in den berühmten mit $E$. Hagen ausgeführten Versuchen mit Wärmestrahlen von $25,5 \mu$ Wellenlänge. Das Ergebnis war, dab sich das Emissionsvermögen aller Metalle mit einer einzigen Ausnahme (Wismut) für diese Strahlen berechnen läbt aus der elektrischen Leitfähigkeit. Bei vielen anderen festen und flüssigen Körpern versagen aber die eïnfachsten Maxwellischen Beziehungen. Es liegt dies daran, dab dio betr. Körper in dem Wellenlängengebiet zwischen 25,5 $\mu$ und den elektrischen Wellen Absorptionserscheinungen zeigen, die durch den molekularen Bau der Materie bedingt sind. Die Maxwellsche Theorie betrachtet die Materie als Kontinuum und kann deshalb diese Einflüsse nicht berücksichtigen. Ein Beispiel für das merkwürdige Verhalten mancher Körper ist z. B. das Wasser, dessen Berechnungsexponent im optischen Gebiet 1,33, im elektrischen Gebiet 9 ist, ohne daß man bis jetzt mit Sicherheit hat feststellen können, wo sich der Übergang vollzieht.

Auf Grund dieser Betrachtungen ist es verständlich, daß Rubens sich immer mit der Aufgabe beschäftigt hat, die Kluft zwischen elektrischer und Wärme der ultraroten Wellen zu überbrücken. Naturgemäß sind zwei Wege möglich, man kann versuchen einerseits die Wellenlänge elelktrisch erzeugter Schwingungen zu verkleinern oder andererseits Wärmestrahlen gröBerer Wellenlänge za isolieren. Beide Wege hat Rubens zu beschreiben versucht. In der Zeit, als er noch an der technischen Hochschule Charlottenburg wirkte, hat er, wie ich aus seinen Gesprächen entnahm, Versuche mit elektrischen Wellen angestellt. Er wollte eine elektrische Entladung durch Metallstüeke aller möglichen Größen hindurch gehen lassen und hoffte auf diese Weise kurze elektrische Wellen zu bekommer. Später hat der Verfasser dieses Berichtes auf seine Veranlassung hin sich längere Zeit mit der Herstellung kurzer elektrischer Wellen beschäftigt. Es gelang auch, den diamaligen., ,Weltrekord'" zu schlagen, indem elektrische Wellen von $2 \mathrm{~mm}$. Wellenlänge nachgewiesen werden konnten. Leider war es unmöglich wegen der Inkonstanz und Launenhaftigkeit der Erscheinung quantitative Messungen auszuführen. Rubens interessierte sich lebhaft für diese Arbeiten, die wegen der erwähnten Schwierigkeiten nicht zu dem erwünschten Abschluß ge- bracht werden konnten. Noch in den letzten Jahren hat er des öfteren die Absicht geäußert, die Versuche fortzuführen. Er erhoffte Erfolge von der Anwendung hohen Gasdruckes, in dem der die Schwingung erzeugende Funke überspringen sollte.

In derselben Zeit, in der ich an den eswähnten Versuchen arbeitete, begann Rubens selbst, wie er sagte, ,den Tunnel an der anderen Seite anzubohren", und zwar mit sehr viel gröBerem Erfolg. Eus gelang ihm in Gemeinschaft mit Wood 1910 bis zu Wellenlängen von etwa $100 \mu$ vorzudringen. Diese Arbeiten waren die Grundlage und die Voraussetzung für das Gelingen der späteren Versuche. Denn hier wurden die experimentellen Methoden zur Isolierung, dem Nachweis und der Messung der Wellenlänge solcher äußerst langwelliger Wärmestrahlen ausgebildet. Zur Absonderung des langwelligen Teiles aus der Gesamtstrahlung eines Areribrenners wurde die sogenannte Quarzlinsenr methode verwendet, die gegenüber den sonst üblichen Prismen oder Gittermethoden den Vor. teil bietet, mit sehr geringem Energieverlust zu arbeiten. Sie beruht darauf, daß Quarz für Wärmestrahlen oberhalb $80 \mu$ Wellenlänge denselben Brechungsexponent wie für elektrische Wellen besitzt, nämlich 2,14 , während für kürzere Wellen der Brechungsexponent dem optischen Wert 1,5 nahe liegt. Außerdem unterstützt die starke Absorption des Quarzes für die kurzwellige Wärmestrahlung die Absonderung der langwelligen.

Zum Nachweis und zur Messung der Energie hatte Rubens das sogenannte Radiomikrometer zu groBer Vollkommenheit ausgebaut. Die Empfindlichkeit dieses. Instrumentes wurde unter Mitarbeit des vortrefflichen Institutsmechanikers Herrn Muselius wesentlich gesteigert, und vor allem die Ruhelage verbessert. Interessant war die Tatsache, daß die geringen, durch Druckänderung der Atmasphäre bedingten Temperaturschwankungen diese Instrumente stark beeinflussen. Luiftdichter AbschluB bewirkt sofort den Fortfall dieser Störung. Zur Wellenlängenmessung wurde ein Interferometer gebaut, zwei Platten aus Quarz, deren Abstand durch eine feine Schraube, genau meBbar, verändert werden konnte.

Dieses Instrumentarium standi im Winter 1910/11 zur Verfügung, als Rubens mir den Vorschlag machte, gemeinsam mit ihm nach Strahlungsquellen $z u$ suchen, die möglichst langwellige Wärmestrahlen aussenden. Theoretische Gesichtspunkte konnten hierbei kaum Fingerzeige geben, in welcher Richtung zu. suchen sei. Sollte ein Temperaturstrahler in Frage kommen, so muBte man jedenfalls Quellen von sehr hoher Temperatur verwenden, da der absolute Wert dez Wärmestrahlungsenergie mit der Temperatur steigt. Sollte die Strahlung eine Luminiszenzstrahlung sein, so war nach den damaligen 
Kenntnissen überhaupt nichts vorauszusagen. Es muBten also auf gut Glück alle möglichen Strahlungsquellen probiert werden. Begonnen wurde mit sehr kräftigen Funken, die von einem Resonanztransformator mit einer Leidener Flaschenbatterie geliefert wurden. Der Lärm, den diese Funkenstrecke erzeugte, war im wahren Sinne des Wortes ohrenbetäubend. Man muBte Watte in die Ohren stopfen, um überhaupt arbeiten zu können. Die ersten Versuche sahen vielversprechend aus. Man bekam einen mächtigen Ausschlag im Radiomikrometer, als die Strahlung der Funkenstrecke durch einen Hohlspiegel in das Radiomikrometer gesandt wurde. Aber leider zeigte es sich sofort, dab die Richtung des Ausschlages nicht eine Temperaturerhöhung, sondern einer Temperaturverminderung entsprach. Wir hatten, wie Rubens lachend meinte, eine ,Kältestrahlung" vor ans. Sehr schnell wurde die wahre Natur dieser Erscheinung geklärt, es waren sehr schnelle Schallschwingungen, die sich ebenso in Lichtwellen durch Hohlspiegel konzentrieren lassen und dann duroh ganz enge Öffnungen hindurehtreten können. Die Wirkung auf das MeBinstrument war die Folge des Schalldruckes, der bei der zufälligen Form des der Stralilung ausgesetzten Teils glücklicherweise eine Drehung nach der ,Kälte-

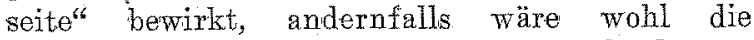
Klärung des. Effektes nicht so schnell erfolgt. Einschalten einer genügenden Quarzoder Papierschicht ließ den Kälteausschlag ver schwinden. Es war aber auch keine Wärmestrahlung da. Alle möglichen Materialien wurden als Elektroden der Funkenstrecke verwendet: nichts. Noch eine ganze Reihe von anderen Strahlungsquellen wurden untersucht, Lichtbogen mit salzgetränkten Kohlen, Lichtbogen zwischen Metallelektroden, Röntgenröhren, Kanalstrahlenröhren. Aber alles umsonst! Nie mehr als' höchistens einigo Zehntel Skalenteilausschlag. Es erschien hoffnungslos. Da: tauchte die Idee auf, die Quarzquecksilberlampe zu probieren. Solche Lampen waren in mehreren Exemplaren im Institut vorhanden, da in dieser Zeit eine Reihe von lichtelektrischen Arbeiten im Gange waren. Rubens hatte kein rechtes $\mathrm{Zu}$ traven mehr auf einen Glückstreffer. Er lieb mich den. Versuch selbst ausführen und verschwand in das Schreibzimmer. Ein schneller Blick ins Fernrohr and ich gehe in das andere Zinmer, wo mich Rubens mit freundlichem, aber spöttischem Lächelin fragt: Nun, sinds 100 Skalenteile? Worauf ich in gewohnter Ruhe antworte: Nein, aber zwanzig!

Sofort unterbricht Rubens seine schriftlichen Arbeiten, und es dauert nur wenige Stunden, bis die neue Strahlung auf ihre Eigenschaften wenigstens im großen und ganzen untersucht ist. Sie wird beim Durchgang durch schwarzes Papier weniger geschwächt wie die Strahlung von $100 \mu$ Wellenlänge, dasselbe ergibt sich sich beim Durch- gang durch amorphen Quarz. Ein Verhalten, das jedenfalls auf das Vorhandensein Iängerer Wellenlängen hindeutet. Die nächsten Wochen werdien nun in intensiver Arbeit der genauen Untersuchung der neuen Strahlung gewidmet. Der Interferometer wird eingeschaltet und damit die Wellenlänge der Strahlung ermittelt.

Es ergab sich, daf die Quarzquecksilberlampe einen ganzen Komplex von Wärmestrahlen verschiedener Wellenlänge aussendet. Durch geeignete Filterung mit Quarz oder schwarzem Karton hießen sich die kürzeren Wellenlängen, die offenbar von der Strahlung der heiBen Quarzwände der Lampe herrühren, aussieben und es blieb eine äuBerst langwellige Strahlung übrig, die wesentliche Intensität bei den Wellenlängen $218 \mu$ und $343 \mu$ aufwies. Damit war mit einem Schlag das Wärmespektrum um mehr wie 11/2 Oktaven erweitert.

Ich übergehe hier die Einzelheiten der weiteren Untersuchangen, die zuerst in zwei $\mathrm{Ab}$ handlungen in den Berichten der preuBischen Akademie der Wissenschaften 1911 niedergelegt sind. Es sei nur. erwähnt, das von der durch $0,4 \mathrm{~mm}$ dicken schwarzen Karton gefilterten Strahlung $37 \%$ der Intensität noch durch einen weiteren solchen Karton hindurch gehen, nach Filterung dureh $5 \mathrm{~cm}$ Quarz sogar $60 \%$, während die Strahlung von 100 wi mur zu $2 \%$ von ebendemselben Karton hindurchgelassen wird.

Von prinzipieller Bedeutung war noch der Nachweis, daß die Strahlung tatsächlich von dem leuchtenden Quecksilberdampf herrührt. Es wurde dies dadurch festgestellt, dab die langwellige Strahlung nach dem Verlöschen der Lampe sofort verschwindet. Späterhin (1913) durfte ich noch an einer Untersuchung teilnehmen, bei der nachgewiesen wurde, dab die Strahlung von $218 \mu$ Wellenlänge von Wasserdampf nur wenig absorbiert wird, die Strahlung von $343 \mu$ Wellenlänge fast gar nicht, ganz im Gegensatz zu den kurzwelligeren Strahlen, die zum Teil in sehr intensiver Weise von Wasserdampf verschluckt werden.

Bej. all diesen Untersuchungen hatte ich Gelegenheit, die Arbeitsweise von Heinrich Rubens aus nächster Nähe kennen zu lernen. Bewundernswert war es, wie er als vielgeplagter Ordinarius der großen. Berliner Universität Zeit und Kraft fand, nachmittags bis in die späte Nacht die Messungsreihen durchzuführen: Dabei erforderten diese Messungen ein ungewöhliches Maß ron Geduld und Ausdaver. Man mul nur bedenken, daB für eine einzige der zahlreichen Interferometerkurven manchmal 100 und mehr Punkte gemessen wurden. Jeder Punkt war wieder das Resultat mindest zweier Einzelmessungen. Dabei waren die Ausschläge der GröBe nach in günstigen Fällen 50 Skalenteile, manchmal aber nur fünf bis zehn Skalenteile, so dal es auf die zehntel Skalenteile ankam. 
Da das Radiomikrometer nie ganz ruhig steht, sondern, abgesehen von kleinen unregelmäBigen Störungen, fast immer einen gleichmäBigen Gang zeigt, wurde der sogenannte ballistische Ausschlag beim Zutritt der Strahlung beobachtet, dann aber noch nach dem Abblendender Strahlung der Rückgang des Ausschlages im Fernrohr verfolgt und aus den so gewonnenen Werten das Mittel genommen. Erst durch diese Ablesungsart lieB sich die enstaunliche Präzision der Messungen erzielen.

Die Isolierung der Wärmestrahlen von $0,34 \mathrm{~mm}$. Wellenlänge gestattete nun; die Maxwellsche Theorie in viel weitgehenderer Weise zu bestätigen, als es früher möglich war. In dem erwähnten Festvortrag von Rubens sind die Exgebnisse seiner Arbeiten in dieser Hinsicht bis 19.17 zusammengestellt. Bei 35 festen Körpern, darunter 20 Kristallen, konnte er den AnschluB an die elektrischen Messungen herstellen, ebenso bei einigen Flüssigkeiten, während bei einer ganzen Reihe von anderen Flussigkeiten; wie Wasser, Alkohol, Glyzerin, die Brücke noch nicht geschlagen werden konnte. Hier sind eben noch Absorptionsgebiete bei noch größeren Wellenlangen vorhanden, wie dies auch schon aus elektrischen Messungen bekannt war.

Jedenfalls erkennt man aus solchen Betrachtungen so recht, welchen Verlust die Physik durch den Tod von Heinrich Rubens erlitten hat, Denn wie kaum ein anderer hat er es verstanden, am ein Gleichnis von Heinrich Hertz zu gebrauchen, Pfeiler auf Pfeiler zu erbauen für das Gewölbe, das sich von den optischen nach den elektrischen Wellen erstrecken sollte, und sicherlich hätte er den SchluBstein in diesem Bau eingefügt, hätte nicht ein bitteres Schicksal den Baumeister vorzeitig abberufen.

\title{
Rubens und die Quantentheorie.
}

\author{
Von J. Franck und R. Pohl, Göttingen.
}

Wenn ein bedeutender Gelehrter aus dem Leben scheidet und seine Schüler und Freunde daran gehen, einen Überblick über sein Lebenswerk zu geben, so ergibt sich oft eine sehr starke Verschiedenheit in der Bewertung der Wichtigkeit der verschiedenen Teilgebiete seiner Forschungstätigkeit. Jeder ist eben geneigt, das als das Wichtigste anzusehen, was seinem eigenen Ideenkreis am nächsten steht. Das geht, wie es das Beispiel von Helmholtz lehrt, manchmal so weit, daB selbst die Disziplin, zu der man den Forscher vor allem zu rechnen wünscht, je nach dem Standpunkt des Berichterstatters verschieden ist. Einen solchen $\mathrm{Z}_{\text {weifel }}$ kann es bei der Besprechung des Lebenswerkes von Heinrich Rubens nicht geben, er war Experimentalphysiker, und zwar ein reiner Experimentalphysiker von höchstem Range. Da mag es denn bei der Durehsicht der Titel der Aufsätze, die in diesern Hefte der "Naturwissenschaften" zu seinem Andenken vereinigt sind, verwunderlich erscheinen, daß die meisten den Namen einer physikalischen Theorie als Utberschrift enthaiten. In der Tat gibt es zwei verschiedene Arten, Experimentalphysik als Forschungsgebiet zu betreiben, die gerade durch ihr Verhältnis zur theoretischen Physik am besten bezeichnet werden. Die eine von ihnen nimmt die Vorstellungsweise der theoretischen Physik insoweit zur Kenntnis, als sie naturgemäls bemüht sein mul, nicht mit ihr in Konflikt zu geraten, sucht aber im übrigen neue Tatsachen auf rein experimentelle Weise aufzufinden, ohne die Absicht, das Einzelexperiment einem größeren theoretischen Gesamtkomplex anzugliedern. Dieser Art der Forschungsrichtung verdanken wir viele unerwartete Entdeckungen, aber sie liefert andererseits auch viel Leerlaufarbeit. Die zweite Art von Experimentaluntersuchungen wird unternommen, um sich mit einer theoretisch begründeten Vorstellungswelt auseinanderzusetzen, sie an Hand der Erfahrung zu prüfen, zu bestätigen oder zu widerlegen. Es könnte scheinen, als ob sie so nur eine Dienerin der theoretischen Physik, eine Wissenschaft geringeren Grades sei, das ist jedoch ein Fehlschlup. Diese Art des experimentellen Forschens folgt micht blind der Fragestellung der Theorie," sondern sie zieht Folgerungen aus ihr, und sie eilt daher meistens der mathematisch-theoretischen Durcharbeitung des: Gebietes weit voraus. Diese. letztere Art der Experimentalphysilk ist es, in der Rubens Meister war, und aus dieser Einstellung entspringt die Verknüpfung seiner Leistungen mit der modernen Vorstellumgswelt der theoretischen Physik, sowohl der Zusammenhang mit der Maxwellschen Theorie, der in einem anderen Artikel geschildert wird, wie auch derjenige mit der Quantentheorie, die zum Teil in Hettners Aufsatz, zum Teil in diesen Zeilen dargestellt werden soll.

Man dxann das Zusammenarbeiten von experimenteller Forschung mit der theoretischen: Anschauwng in der Quantentheorie geradezu als ein klassisches Beispiel dafür betrachten, wie sehr diese beiden $Z$ weige unserer $W$ issenschaft aufeinander angewiesen sind und sich gegenseitig bei harmonischem Zusammenwirken ergänzen. $\mathrm{Da}$ ist zuerst an die Patenschaft der Experimentatoren, von denen Rubens einer war, bei der Aufstellung der Strahlungsgleichung des schwarzen Körpers zu denken (siehe Hettner und das Planckheft $\left.{ }^{1}\right)$ ). Es genïgt daher hier, den $\mathrm{Zu}$ -

1) Diese Zeitschrift S. 1033. 